Research Article

\title{
Unit Root Analysis Method of Actual Crack Behavior of the Concrete Dam Based on Residuals of the Monitoring Model
}

\author{
Bo Xu $\left(\mathbb{D},{ }^{1}\right.$ Zhanchao Li, ${ }^{1}$ and Jinlei Zhao ${ }^{2}$ \\ ${ }^{1}$ College of Hydraulic Science and Engineering, Yangzhou University, Yangzhou 225009, China \\ ${ }^{2}$ College of Water-conservancy and Hydropower, Hohai University, Nanjing 210098, China \\ Correspondence should be addressed to Bo Xu; xubo@yzu.edu.cn
}

Received 26 November 2019; Revised 23 June 2020; Accepted 15 September 2020; Published 24 September 2020

Academic Editor: Guoyang Fu

Copyright $\odot 2020$ Bo Xu et al. This is an open access article distributed under the Creative Commons Attribution License, which permits unrestricted use, distribution, and reproduction in any medium, provided the original work is properly cited.

Analysis on the actual crack behavior is of important significance to evaluate safety of the concrete dam. In this paper, mainly based on the research status on cracks of the concrete dam, the unit root analysis method of actual crack behavior of concrete dam is proposed based on the monitoring data and autoregression model, aiming to enrich the analysis methods of crack behavior of concrete dam and deepen the understanding of crack behavior of concrete dam. Firstly, the feasibility of analyzing actual crack behavior based on residual sequence of the crack opening displacement monitoring model is discussed. Next, the basic principle of the unit root analysis method of crack behavior is elaborated and the unit root analysis method of actual crack behavior is established by studying prior conditions of the autoregression model and parameter estimation methods of the autoregression model, as well as determination criteria of the model order. Finally, the proposed unit root analysis method of actual crack behavior is applied to analyze monitoring data of a concrete gravity-arch dam. The result shows that the unit root analysis method of actual crack behavior is relatively simple and convenient in practical application, which is an available method to evaluate crack behavior based on data in dam health monitoring.

\section{Introduction}

Loads on the concrete dam such as hydraulic pressure and temperature loads vary continuously during the service period, thus resulting in the change of crack behavior. In some cases, the crack may expand along the length and (or) depth upon disadvantageous load combination, which means the crack behavior will change to some extent. Analyzing the actual crack behavior is of important significance to evaluate safety of the concrete dam. Therefore, it is very necessary to study the analytical methods of crack behavior.

A large number of scholars have explored the crack development law of concrete structure through numerical simulation and model test and have achieved a lot of beneficial results. $\mathrm{Wu}$ et al. [1] analyzed the whole process of I-II composite crack propagation by numerical simulation. Sukumar et al. [2] introduced the fast tracking method into the extended finite element system and simulated the coplanar multifracture propagation problem. $\mathrm{Zi}$ et al. [3] simulated the dynamic crack propagation by extended finite element and proved by actual cases that the extended finite element could capture the mixed cracking under impacts in experiment. Zhang et al. [4] analyzed the initial crack propagation of Koyna gravity dam under the seismic loads based on the extended finite element method and model test. Dias et al. [5] simulated the crack propagation process of a concrete gravity dam by crack-path-field and strain injection techniques. Chiou et al. [6] simulated the crack propagation on plane successfully through the manifold element method. Pan et al. [7] made a contrast analysis on cracking behaviors and crack propagation process of the Koyna gravity dam and Dagangshan arch dam under the seismic loads by the extended finite element method with cohesive constitutive relations, the crack band finite element method with plastic-damage relations, and the finite element Drucker-Prager elasto-plastic model. Hariri-Ardebil [8] discussed effects of foundation nonlinearity on crack propagation of concrete dam by the numerical simulation method. Hariri-Ardebil and Seyed-Kolbadi [9] analyzed crack 
behaviors of concrete gravity dam, buttress dam, and arc dam under the seismic loads by the smeared crack model and verified the results through experiments. Hariri-Ardebil et al. [10-12] also studied damage occurrence, intensity, location, number, size, and propagation pattern of concrete dams, explored potential failure modes identification and quantification of concrete dams subjected to seismic excitation, and investigated seismic response of a coupled dam-reservoirfoundation system considering pressure effects at opened joints. Shi et al. [13] simulated the crack propagation process of a gravity dam based on the cohesive crack model by using the polygon proportional boundary finite element method. Wang et al. [14] analyzed crack expansion angle and length under hydraulic pressure by the XFEM-FVM model with consideration to liquid-solid coupling. Shi et al. [15] carried out a simulation analysis on multiple discrete cracks in concrete dams based on the extended virtual crack model and analyzed the expansion processes and interactions of cracks. The above numerical simulation methods mainly analyze crack behavior based on mechanical numerical models, and the results are beneficial to the understanding and analysis of engineers. The key of this kind of methods is to construct the mechanical numerical models reflecting the actual structure. However, due to the complexity of the actual dam structural system, the corresponding mechanical numerical models are often difficult to construct. The model test methods simplify the dam structure system and its external conditions. So, they are difficult to analyze the complex phenomenon of actual projects. Furthermore, cost of model test methods is usually very high.

At the same time, some mathematical methods are applied more and more to analyze crack propagation. Based on the theory of dissipative structure and entropy theory, Cong et al. [16] studied the concrete cracking process from the perspective of the energy distribution state. Wu et al. [17] proposed an analytical method of concrete dam crack generation and development based on the entropy theory and verified its feasibility through an engineering case. Li et al. [18] studied the time-varying law of concrete dam cracks by combining wavelet analysis and phase-space reconstruction. Bernstone and Heyden [19] put forward the analytical method of crack propagation of concrete structure in hydropower stations based on the image analysis and verified the feasibility of this method through engineering cases. Li et al. [20] proposed the online diagnosis method for diagnostic analysis of abnormal values during the development of concrete dam cracks. This kind of methods is essentially phenomenological analyses based on data, which are often unable to provide analytical results with mechanics mechanism. However, they can be applied to analyze actual projects, which are helpful to deepen the understanding of the actual phenomena, especially for the complex phenomena.

Based on the above analysis, numerical simulation, model test, and mathematical method are main analytical methods of crack behavior. The analytical method proposed in this paper belongs to the third kind. The main reasons for adopting this method are as follows. The mathematical method can analyze the crack behavior of concrete dams according to monitoring data. Monitoring data of concrete dams are equal to $1: 1$ model test, which can reflect the operating status of concrete dams most directly and effectively. So, analyzing the crack behavior based on monitoring data is the analytical method closest to the actual engineering situations. However, there are relatively few mathematical methods that analyze crack behavior of concrete dams according to monitoring data. Therefore, further research studies on the analytical methods of crack behavior of concrete dams based on monitoring data are needed.

In this paper, the unit root analysis method of actual crack behavior of the concrete dam is constructed based on monitoring data and related theory of the autoregression model, in the framework of structural health monitoring. It is aiming to enrich the analysis methods of crack behavior, deepen the understanding of crack behavior of the concrete dam, and lay foundations for evaluating safety of the concrete dam. The main content of this paper includes

(1) Feasibility of analyzing actual crack behavior based on residuals of the crack opening displacement monitoring model

(2) Basic principles of the unit root analysis method of crack behavior

(3) Establishment of the unit root analysis method

(4) Application of unit root analysis method in an actual project

\section{Feasibility of Analyzing Actual Crack Behavior Based on Residuals of the Monitoring Model}

Changes of crack opening displacement generally include time effect component caused by time effect factors, temperature component caused by periodic fluctuation of temperature changing with seasons, and water pressure component caused by fluctuations of reservoir water level, as well as the random changes caused by other factors [21,22]. Significant changes of state of concrete dam cracks will be manifested on large violations of the observation data curve of crack opening displacement or variation trends of water pressure, temperature, and time effect components during modeling analysis based on the monitoring model. However, crack propagation along the depth and (or) length will cause changes of the crack opening displacement. Influences of such crack propagation on crack opening displacement generally cannot be reflected by water pressure component, temperature component, or time effect component in monitoring model analysis, but are included in the residuals of the monitoring model.

Abnormalities of concrete dam cracks are mainly manifested on changes of the crack depth and (or) length, thus causing changes of the crack opening displacement. In other words, the crack opening displacement CMOD under normal circumstances includes two parts:

$$
\mathrm{CMOD}=\mathrm{CMOD}_{0}+\mathrm{CMOD}_{h}
$$

where $\mathrm{CMOD}_{0}$ is the initial value of crack opening displacement and $\mathrm{CMOD}_{h}$ is changes of crack opening 
displacement caused by variations of water pressure, temperature, and time effect.

The crack opening displacement is composed of three parts upon crack abnormality:

$$
\mathrm{CMOD}_{\text {un }}=\mathrm{CMOD}_{0}+\mathrm{CMOD}_{h}+\mathrm{CMOD}_{j},
$$

where $\mathrm{CMOD}_{\text {un }}$ is crack opening displacement upon crack abnormality, $\mathrm{CMOD}_{0}$ is the initial value of crack opening displacement, $\mathrm{CMOD}_{h}$ is changes of crack opening displacement caused by variations of water pressure, temperature, and time effect, and $\mathrm{CMOD}_{j}$ is changes of crack opening displacement caused by variations of crack length and (or) depth.

According to equations (1) and (2), if $\mathrm{CMOD}_{0}$ is fixed and $\mathrm{CMOD}_{h}$ remains constant under the same loads before and after crack abnormality, the crack opening displacement changes after the crack abnormality are mainly reflected by $\mathrm{CMOD}_{j}$ caused by variations of crack depth and (or) length. If the crack opening displacement is $\mathrm{CMOD}_{i}, \mathrm{CMOD}_{j}$ is

$$
\mathrm{CMOD}_{j}=\mathrm{CMOD}_{i}-\left(\mathrm{CMOD}_{0}+\mathrm{CMOD}_{h}\right) \text {, }
$$

where $\mathrm{CMOD}_{j}$ does not exist at crack stability, but exists at crack abnormality. Since $\mathrm{CMOD}_{i}$ is the measured value and $\mathrm{CMOD}_{0}$ is the initial value of crack opening displacement and both are known, $\mathrm{CMOD}_{j}$ can be calculated after $\mathrm{CMOD}_{h}$ is calculated. According to the monitoring model of crack opening displacement in the concrete dam, the crack opening displacement caused by variations of water pressure $H$, temperature $T$, and time effect $\theta$ can be expressed as follows:

$$
\mathrm{CMOD}_{h}=f(H, T, \theta) .
$$

The crack opening displacement in the concrete dam can be analyzed by the statistical model, deterministic model, and hybrid model $[21,23]$. The statistical model can accurately measure the correlation degree and regression fitting degree of each factor, which is more simple and convenient to analyze the influence of multiple factors. A lot of literature studies show that the crack opening displacement in the concrete dam can be well analyzed by the statistical model $[20,24-26]$. Therefore, the statistical model is adopted to analyze the crack opening displacement of the concrete dam in this paper.

When the crack opening displacement in the concrete dam is analyzed by the statistical model, equation (4) can be expressed as follows:

$$
\mathrm{CMOD}_{h I}=\sum_{k=0}^{m_{1}} a_{k} H^{k}+\sum_{k=1}^{m_{2}} b_{k} T_{k}+c_{1} \theta+c_{2} \ln \theta+\varepsilon,
$$

where $a_{k}, b_{k}, c_{1}$, and $c_{2}$ are regression coefficients. $H$ is water pressure. $m_{1}$ is the number of water pressure factors or the highest power of water pressure factors. $T_{k}$ is the change in the measured value of each thermometer. $m_{2}$ is the number of temperature measuring points. $\theta$ is calculated from the first day of each stage. It is in units of 100 days and increases by 0.01 for every additional day. $\varepsilon$ is the residuals.
It has to point out that there are other options of expressions of temperature or time effect factors according to monitoring data. When temperature monitoring data is lacking, the temperature component can be expressed as a periodic function. Time effect component can also be expressed as a polynomial. In this case, equation (4) can be expressed as follows:

$$
\begin{aligned}
\mathrm{CMOD}_{h I I}= & \sum_{k=0}^{m_{1}} a_{k} H^{k}+b_{1} \sin S+b_{2} \cos S+b_{3} \sin S \cos S \\
& +b_{4} \sin ^{2} S+\sum_{k=1}^{m_{3}} c_{k} \theta^{k}+\varepsilon
\end{aligned}
$$

where $b_{1}, b_{2}, b_{3}, b_{4}$, and $c_{k}$ are regression coefficients. $m_{3}$ is the number of terms of the polynomial. $S=(2 \pi k t / 365)$ when the cycle is a year or half a year, $k$ is 1 or 2 . And $t$ is the number of days counted from the initial monitoring date. The rest symbols have the same meaning as those in equation (5).

Substitute equation (5) into equation (3), and it can be concluded that

$$
\begin{aligned}
\mathrm{CMOD}_{j}= & \mathrm{CMOD}_{i}-\left(\mathrm{CMOD}_{0}+\sum_{k=0}^{m_{1}} a_{k} H^{k}+\sum_{k=1}^{m_{2}} b_{k} T_{k}\right. \\
& \left.+c_{1} \theta+c_{2} \ln \theta+\varepsilon\right) .
\end{aligned}
$$

The above equation can be rewritten as follows:

$$
\begin{aligned}
\mathrm{CMOD}_{j}+\varepsilon= & \mathrm{CMOD}_{i}-\left(\mathrm{CMOD}_{0}+\sum_{k=0}^{m_{1}} a_{k} H^{k}\right. \\
& \left.+\sum_{k=1}^{m_{2}} b_{k} T_{k}+c_{1} \theta+c_{2} \ln \theta\right) .
\end{aligned}
$$

Let $c \bmod _{j}=\mathrm{CMOD}_{j}+\varepsilon$; then,

$$
\begin{aligned}
c \bmod _{j}= & \mathrm{CMOD}_{i}-\left(\mathrm{CMOD}_{0}+\sum_{k=0}^{m_{1}} a_{k} H^{k}+\sum_{k=1}^{m_{2}} b_{k} T_{k}\right. \\
& \left.+c_{1} \theta+c_{2} \ln \theta\right) .
\end{aligned}
$$

When the monitoring model is equation (6), $c \bmod _{j}$ can be expressed as follows:

$$
\begin{aligned}
c \bmod _{j}= & \mathrm{CMOD}_{i}-\left(\mathrm{CMOD}_{0}+\sum_{k=0}^{m_{1}} a_{k} H^{k}+b_{1} \sin S\right. \\
& \left.+b_{2} \cos S+b_{3} \sin S \cos S+b_{4} \sin ^{2} S+\sum_{k=1}^{m_{3}} c_{k} \theta^{k}\right) .
\end{aligned}
$$


$\operatorname{cmod}_{j}$ only contains $\varepsilon$, but no $\mathrm{CMOD}_{j}$ before crack abnormality. However, $c \bmod _{j}$ contains $\varepsilon$ and $\mathrm{CMOD}_{j}$ after crack abnormality. The probability distribution pattern will be changed accordingly.

Therefore, residual sequence of the crack opening displacement monitoring model can indirectly reflect the crack abnormality, which is time series, and can be used to analyze the crack behavior. In this paper, combined with the above analysis, the variation of crack behavior is mapped to the stability of residual sequence of the crack opening displacement monitoring model. Unit root analysis is one of the methods to study the stability of time series. In this paper, it is applied to the analysis of crack behavior of the concrete dam, and the basic principles are described below.

\section{Basic Principles of Unit Root Analysis Method}

Stability of data sequence can be analyzed by judgment and detection of smoothness of data sequence. Smoothness detection methods of data sequence include scatter diagram, autocorrelation function method, and unit root method. Among them, the unit root method is relatively accurate [27]. In this paper, the unit root method is used to analyze smoothness of residual sequence of the crack opening displacement monitoring model. If the residual sequence is smooth, the crack behavior is stable; otherwise, the crack may expand. Furthermore, crack propagation possibility at different periods can be analyzed by analyzing smoothness of residual sequences of the crack opening displacement monitoring models, thus enabling to analyze the development of crack behavior.

Considering the following time sequence model,

$$
y_{t}=a y_{t-1}+\varepsilon_{t}
$$

where $a$ is coefficient and $\varepsilon_{t}$ is the white noise, $\varepsilon_{t} \sim\left(0, \sigma^{2}\right) \cdot y_{t}$ is a time sequence.

The model corresponding to equation (11) is the 1-order autoregression model. The mean, variance, and covariance of this model can be calculated by

$$
\left\{\begin{array}{l}
E\left(y_{t}\right)=0, \\
\operatorname{var}\left(y_{t}\right)=\frac{\sigma^{2}}{1-a^{2}}, \\
\operatorname{cov}\left(y_{t}, y_{t+k}\right)=\frac{\sigma^{2} a^{k}}{1-a^{2}} .
\end{array}\right.
$$

If $|a|<1, y_{t}$ meets three conditions of weak equilibrium: both the mean and the variance are constants and the covariance is unrelated with time, then $y_{t}$ is smooth time sequence, otherwise $y_{t}$ is not.

Equation (11) can be rewritten as follows:

$$
\left\{\begin{array}{l}
y_{t}-a y_{t-1}=\varepsilon_{t} \\
\text { or }(1-\mathrm{a} L) y_{t}=\varepsilon_{t},
\end{array}\right.
$$

where $L$ is the lag operator and its characteristic equation is $A(z)=1-a z=0$, which has only one $\operatorname{root} z=(1 / a)$. When $|a|<1, y_{t}$ is smooth time sequence. In other words, $y_{t}$ is smooth time sequence when $|z|>1$.

Generally, the $p$-order autoregression model is recorded as $\operatorname{AR}(p)$ :

$$
y_{t}=a_{1} y_{t-1}+a_{2} y_{t-2}+\cdots+a_{p} y_{t-p}+\varepsilon_{t} .
$$

Its characteristic equation is

$$
1-a_{1} z-a_{2} z^{2}-\cdots-a_{p} z^{p}=0 .
$$

It can be proved that if absolute values of all roots of the characteristic equation are larger than 1; in other words, when all roots are out of the unit circle, the model of equation (14) is stable, otherwise, it is not. Besides, if the characteristic equation has one root on the unit circle, $y_{t}$ has one unit root. Stability of $y_{t}$ can be analyzed by testing the unit root of the characteristic equation $(15)$ [28, 29].

The $p$-order autoregression model corresponding to equation (14) can be rewritten as follows:

$$
y_{t}=\sum_{j=1}^{p} a_{j} y_{t-j}+\varepsilon_{t}, \quad t \in Z
$$

where $\varepsilon_{t} \sim\left(0, \delta^{2}\right)$ is the white noise with 0 mean and $\delta^{2}$ variance. $a_{1}, a_{2}, \ldots, a_{p}$ are autoregression coefficients of the $\operatorname{AR}(p)$ model. If $a_{1}, a_{2}, \ldots, a_{p}\left(a_{p} \neq 0\right)$ make the zero points of $A(z)$ outside the unit circle, the time sequence $y_{t}$ is smooth. In other words, to ensure that $y_{t}$ is a smooth sequence, $A(z)$ has to meet the following condition:

$$
A(z)=1-\sum_{j=1}^{p} a_{j} z^{j} \neq 0, \quad|z| \leq 1 .
$$

Equation (16) can be rewritten by the lag operator $L$ :

$$
A(L) y_{t}=\varepsilon_{t} \text {. }
$$

Suppose $A(z)$ has $k$ different roots $z_{1}, z_{2}, \ldots, z_{k}$. For $1<\rho<\min \left\{\left|z_{j}\right|\right\}, A^{-1}(z)=(1 / A(z))$ is the analytic function in $\{z:|z| \leq \rho\}$. Therefore, there is a Taylor series:

$$
A^{-1}(z)=\sum_{j=0}^{\infty} \varphi_{j} z^{j}, \quad|z| \leq \rho .
$$

Thus, the following equation is true:

$$
y_{t}=A^{-1}(L) A(L) y_{t}=A^{-1}(L) \varepsilon_{t}=\sum_{j=0}^{\infty} \varphi_{j} \varepsilon_{t-j} .
$$

Suppose the smooth sequence $y_{t}$ has an autocovariance function $v_{k}$. If there is a nonnegative function $f(\lambda)$ on the $[-\pi, \pi]$ that makes the following equation true,

$$
v_{k}=\int_{-\pi}^{\pi} f(\lambda) e^{i k \lambda} \mathrm{d} \lambda, \quad k \in Z,
$$

$f(\lambda)$ is the spectral density function of $y_{t}$ or $v_{k}$. According to the Hergoltz theorem, the spectral density function of the smooth time sequence is sole [30]. 
According to equations (20) and (21), the spectral density function of the $\operatorname{AR}(p)$ sequence $y_{t}$ is

$$
f(\lambda)=\frac{\sigma^{2}}{2 \pi}\left|\sum_{j=0}^{\infty} \varphi_{j} e^{i k \lambda}\right|^{2}=\frac{\sigma^{2}}{2 \pi\left|A\left(e^{i \lambda}\right)\right|^{2}},
$$

where $A(z)$ is the characteristic polynomial of the $\operatorname{AR}(p)$ model corresponding to equation (16). Equation (22) shows that $f(\lambda)$ is a positive even function. If $A(z)$ has complex roots $z_{j}=\rho_{j} e^{i \lambda_{j}}$ close to the unit circle, $f(\lambda)$ will have a peak value around $\lambda_{j} . f\left(\lambda_{j}\right) \longrightarrow \infty$ when $\rho_{j} \longrightarrow 1$, and it indicates that $y_{t}$ will become not smooth. In fact, equation (16) has no smooth solution when $A(z)$ has a root on the unit circle.

According to equation (22), the time sequence becomes not smooth from smooth when $A(z)$ has complex roots $z_{j}=$ $\rho_{j} e^{i \lambda_{j}}$ approaching to the unit circle. Based on this theory, stability of the residual sequence of the crack opening displacement monitoring model can be analyzed by variations of the distance between roots of characteristic polynomial $A(z)$ of the autoregression model of residuals of the crack opening displacement monitoring model and the unit circle. On this basis, the crack behavior can be analyzed.

For crack monitoring data at different periods, autoregression models can be established based on the residual sequences of the crack opening displacement monitoring models. Then, roots of characteristic polynomial $A(z)$ of the autoregression models can be calculated. If roots of $A(z)$ are far away from the unit circle, the crack tends to be stable. If roots of $A(z)$ approach to the unit circle gradually, the crack tends to be unstable. When roots of $A(z)$ are on or in the unit circle, the crack may expand. Therefore, the development of crack behavior can be obtained according to variations of the roots of characteristic polynomial $A(z)$ at different periods.

\section{Construction of Unit Root Analysis Method for Crack Behavior}

4.1. Prior Conditions of the $A R(p)$ Model Establishment. A monitoring model is established with the crack monitoring data that need to be analyzed and the residual sequence can be obtained. Before the establishment of the $\mathrm{AR}(p)$ model, it has to test whether the residual sequence meets the prior conditions of the $\operatorname{AR}(p)$ model. There are two prior conditions of the $\operatorname{AR}(p)$ model: (1) the autocorrelation coefficient of residual sequence of the crack monitoring model has the trailing property and (2) partial autocorrelation coefficient of residual sequence of the crack monitoring model has the truncation property. Trailing property means that the autocorrelation coefficient decays exponentially. Truncation property means that the partial autocorrelation coefficient is equal to 0 after a certain number of delayed steps or a certain number of orders.

If the residual sequence of the crack monitoring model is $x_{t}$, the autocorrelation coefficient of residual sequence is

$$
\gamma_{k}=\frac{\sum_{t=1}^{n-k}\left(x_{t}-\bar{x}\right)\left(x_{t+k}-\bar{x}\right)}{\sum_{t=1}^{n}\left(x_{t}-\bar{x}\right)^{2}},
$$

where $\gamma_{k}$ is the autocorrelation coefficient of the residual sequence, $k=0,1,2,3, \ldots n$ is the total number of residual samples. $\bar{x}$ is sample mean, and the calculation formula of $\bar{x}$ is

$$
\bar{x}=\frac{1}{n} \sum_{t=1}^{n} x_{t}
$$

Partial autocorrelation coefficient of residual sequence of the crack monitoring model is

$$
\left(\begin{array}{c}
\varphi_{1} \\
\varphi_{2} \\
\vdots \\
\varphi_{k}
\end{array}\right)=\left(\begin{array}{cccc}
1 & \gamma_{1} & \cdots & \gamma_{k-1} \\
\gamma_{1} & 1 & \cdots & \gamma_{k-2} \\
\vdots & \vdots & \ddots & \vdots \\
\gamma_{k-1} & \gamma_{k-2} & \cdots & 1
\end{array}\right)^{-1}\left(\begin{array}{c}
\gamma_{1} \\
\gamma_{2} \\
\vdots \\
\gamma_{k}
\end{array}\right),
$$

where $\varphi_{i}$ is the partial autocorrelation coefficient, $i=1,2,3, \ldots, k$.

4.2. Establishment of the Autoregression Model. Estimation of the model coefficients and calculation of order number are two problems that have to be solved to establish the autoregression model of residual sequence of the crack monitoring model. Yule-Walker estimation is the simplest and widely used parameter estimation method of the autoregression model [31-33]. In this paper, Yule-Walker estimation is applied to estimate parameters for the $\operatorname{AR}(p)$ model with residual sequence $x_{1}, x_{2}, \ldots, x_{N}$.

The autocovariance function $\widehat{v_{k}}$ can be estimated by residual sequence of the crack monitoring model:

$$
\widehat{v_{k}}=\frac{1}{N} \sum_{i=1}^{N-k}\left(x_{i}-\mu\right)\left(x_{i+k}-\mu\right), \quad k=0,1,2, \ldots, p,
$$

where $\mu=(1 / N) \sum_{i=1}^{N} x_{i}$.

For the $\operatorname{AR}(p)$ model, its autocovariance function $v_{0}, v_{1}, \ldots, v_{p}$ satisfies the following condition:

$$
\left[\begin{array}{c}
v_{1} \\
v_{2} \\
\vdots \\
v_{p}
\end{array}\right]=\left[\begin{array}{cccc}
v_{0} & v_{1} & \cdots & v_{p-1} \\
v_{1} & v_{0} & \cdots & v_{p-2} \\
\vdots & \vdots & \ddots & \vdots \\
v_{p-1} & v_{p-2} & \cdots & v_{0}
\end{array}\right]\left[\begin{array}{c}
a_{1} \\
a_{2} \\
\vdots \\
a_{p}
\end{array}\right] .
$$

According to the Yule-Walker equation in (27), the calculation formulas of moment estimation of autocovariance coefficients $\widehat{a_{1}}, \widehat{a_{2}}, \ldots, \widehat{a_{p}}$ and the moment estimation of the white noise variance $\widehat{\sigma}^{2}$ are as follows: 


$$
\begin{aligned}
& {\left[\begin{array}{c}
\widehat{a_{1}} \\
\widehat{a_{2}} \\
\vdots \\
\widehat{a_{p}}
\end{array}\right]=\left[\begin{array}{cccc}
\widehat{v_{0}} & \widehat{v_{1}} & \cdots & \widehat{v_{p-1}} \\
\widehat{v_{1}} & \widehat{v_{0}} & \cdots & \widehat{v_{p-2}} \\
\vdots & \vdots & \ddots & \vdots \\
\widehat{v_{p-1}} & \widehat{v_{p-2}} & \cdots & \widehat{v_{0}}
\end{array}\right]^{-1}\left[\begin{array}{c}
\widehat{v_{1}} \\
\widehat{v_{2}} \\
\vdots \\
\widehat{v_{p}}
\end{array}\right], } \\
& \widehat{\sigma}^{2}=\widehat{v_{0}}-\sum_{i=1}^{p} \widehat{a_{i}} \widehat{v_{i}} .
\end{aligned}
$$

When $p$ is relatively large, the autoregression coefficients and the white noise variance can be calculated by recursive algorithms, such as Levinson algorithm [34] and Durbin algorithm [35]. In this paper, Levinson algorithm is used.

Since the order number $p$ of autoregression model is generally unknown, the order number needs to be estimated. In practical application, AIC criterion [36], BIC criterion [37], and HQIC criterion [38] are common methods to determine the order number. If the order number of the AR model of residual sequence of the crack monitoring model is $k$, variance $\hat{\sigma}_{k}^{2}$ of the fitting residual of the corresponding $\mathrm{AR}(k)$ model can be calculated, and then the order number of the model can be determined by $\widehat{\sigma}_{k}^{2}$ according to the AIC criterion, BIC criterion, and HQIC criterion.

\section{(1) AIC criterion function is}

$$
\operatorname{AIC}(k)=\ln \left(\widehat{\sigma}_{k}^{2}\right)+\frac{2 k}{n}, \quad k=0,1,2, \ldots, p_{0}
$$

where $n$ is the number of residual samples and $p_{0}$ is the upper limit of model order number. The minimum $k$ corresponding to the minimum $\operatorname{AIC}(k)$ is the order number of the $\operatorname{AR}(p)$ model determined by AIC criterion.

(2) BIC criterion function is

$$
\mathrm{BIC}(k)=\ln \left(\widehat{\sigma}_{k}^{2}\right)+\frac{k \ln (n)}{n},
$$

where signs are the same with those in equation (29). The minimum $k$ corresponding to the minimum $\operatorname{BIC}(k)$ is the order number of the $\operatorname{AR}(p)$ model determined by BIC criterion.

(3) QHIC criterion function is

$$
\mathrm{QHIC}(k)=\ln \left(\widehat{\sigma}_{k}^{2}\right)+2 \frac{k \ln (\ln (n))}{n},
$$

where signs are the same with those in equation (29). The minimum $k$ corresponding to the minimum $\mathrm{QHIC}(k)$ is the order number of the $\mathrm{AR}(p)$ model determined by QHIC criterion.

In this paper, the order number $p$ of the $\operatorname{AR}(p)$ model is determined by the above three criteria. If $p$ determined by the above three criteria is the same, it is used as the order number of the autoregression model, and the characteristic polynomial of the autoregression model is established according to this $p$ directly. If $p$ determined by the above three criteria is different, the characteristic polynomials of the autoregression model shall be established according to different $p$.

After order number and coefficients of the autoregression model of residual sequence of the crack monitoring model are obtained, the characteristic polynomial of the autoregression model can be constructed; thus, the corresponding characteristic equation is also established. If the order number of the autoregression model of residual sequence is $p$, the corresponding characteristic equation is

$$
1-\sum_{j=1}^{p} a_{j} z^{j}=0
$$

The roots $z_{j}$ of the characteristic equation can be calculated with equation (32). And the minimum root can be obtained:

$$
C_{\varepsilon}=\left|\min \left(z_{j}=\rho_{j} e^{i \lambda_{j}}\right)\right|,
$$

where $C_{\varepsilon}$ is the minimum root of the characteristic equation of the $\operatorname{AR}(p)$ model. $z_{j}=\rho_{j} e^{i \lambda_{j}}$ are all roots of (32)fd32, including possible repeated roots, $j=1,2, \ldots, p$.

The relationship between the minimum root of the characteristic equation and the unit circle, namely, the relationship between $C_{\varepsilon}$ and 1 , is examined. If $C_{\varepsilon} \leq 1$, the residual sequence of the crack monitoring model is not stable and the crack may expand. Otherwise, the residual sequence of the crack monitoring model is stable and no crack abnormality occurs. On this basis, the process curve of the minimum roots at different periods can be drawn, which can be used to analyze the crack behavior.

The flow chart of the unit root analysis method is shown in Figure 1. On this basis, the crack behavior can be analyzed reasonably.

\section{Engineering Case}

5.1. Project Profile. The dam of a hydropower station is a concrete solid gravity-arc dam with a crest elevation of $233.0 \mathrm{~m}$. The maximum dam height and the total crest length are $110 \mathrm{~m}$ and $525 \mathrm{~m}$, respectively. It is divided into 28 dam sections. The normal storage water level and storage capacity of the reservoir are $223.0 \mathrm{~m}$ and $26.12 \times 10^{8} \mathrm{~m}^{3}$, respectively. The design flood level $(0.1 \%)$ is $227.20 \mathrm{~m}$ and the check flood level $(0.02 \%)$ is $229.20 \mathrm{~m}$. It is an incomplete yearly regulation reservoir. The main construction of the hydropower station began in March, 1985, and the reservoir started saving water in 1992. The completion acceptance of the project was in December, 1996. The unit root analysis method of actual crack behavior is applied to analyze monitoring data of a crack measuring point in this concrete gravity-arch dam from January, 2002, to March, 2009. The measured data curve of the crack opening displacement is shown in Figure 2. The variation curve of the measured upper water level in the concrete gravity-arc dam is shown in Figure 3. 


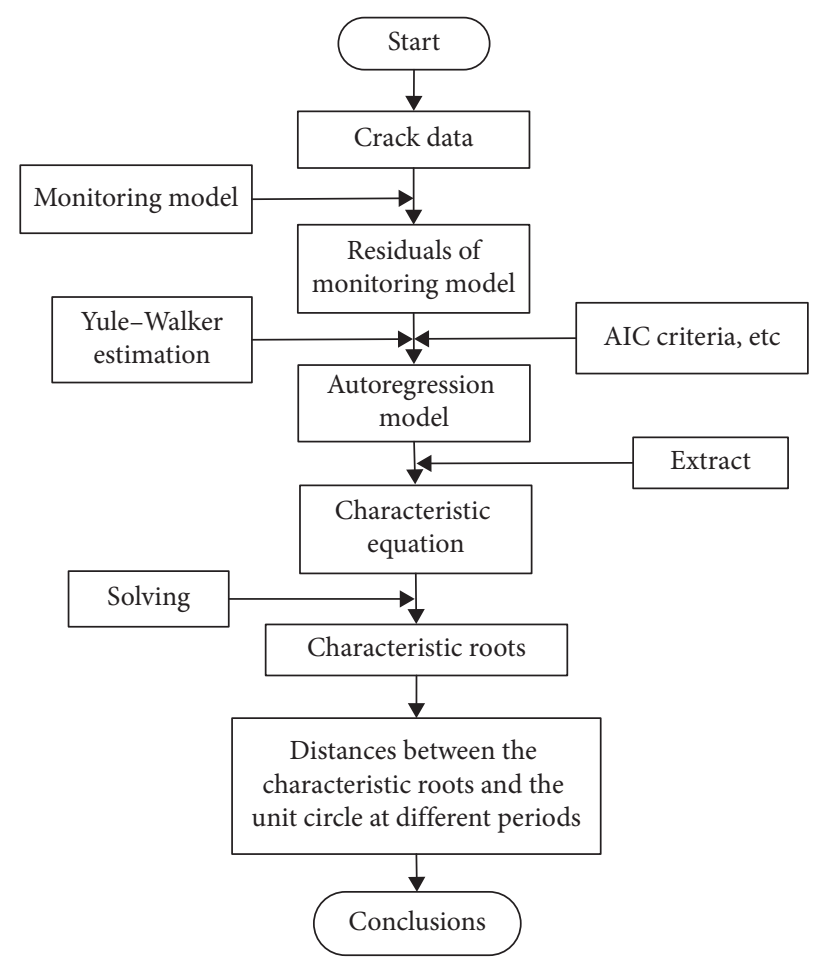

FIgURE 1: Flow chart of the unit root analysis method.

5.2. Calculation and Analysis. In order to analyze the development of crack behavior at different periods, monitoring data of crack opening displacement in the concrete dam can be divided according to the operational control conditions or specified time scale [27]. On this basis, the monitoring data can be analyzed by the segment modeling method, and the residual sequences of monitoring models at different periods can be obtained. In this case study, the specified time scale is one year. It is feasible to segment according to other time scales, and the analysis method after segmentation is the same as that in this paper.

The temperature monitoring data is lacking, so the monitoring model in equation (6) can be adopted. Measured data of crack opening displacement are analyzed yearly. According to [20-22], monitoring models of crack opening displacement are established based on measured data from 2002 to 2008, and the corresponding residual sequences of the monitoring models are obtained, which are shown in Figure 4.

The residual sequence of the monitoring model in 2002 is analyzed in detail and the residual sequences of monitoring models in rest years are analyzed in the same way. Autocorrelation coefficient and partial autocorrelation coefficient of the residual sequence of the monitoring model in 2002 are calculated by the self-compiled program, and results are shown in Figures 5 and 6. It can be seen that the autocorrelation coefficient of residual sequence in 2002 has the trailing property and the partial autocorrelation coefficients has the truncation property. The residual sequence meets the prior conditions of the autoregression model; therefore, it can be analyzed by the autoregression model.
Suppose the order number of the model is $k$ and the model parameters are estimated by the Yule-Walker estimation method. The order number $p$ of $\operatorname{AR}(p)$ model is determined according to the AIC criterion, BIC criterion, and HQIC criterion together. Variations of AIC, BIC, and HQIC values with $k$ are shown in Figures 7-9.

It can be seen from Figures 7-9 that minimum AIC, BIC, and HQIC values are achieved when $k=4$ and $k=6$ and the two minimum values are very close. Therefore, it is impossible to determine the order number $p$ directly. In other words, $p$ may be 4 or 6 . Hence, the 4 -order and 6-order autoregression models have to be established, and two different characteristic polynomials are obtained. Characteristic roots of the characteristic equations are calculated, and the results are shown in Table 1 .

Table 1 shows that the characteristic roots of the 4-order characteristic equation are $1.54,1.94,1.94$, and 1.07. The minimum root is 1.07 , which is greater than 1.0. The characteristic roots of the 6-order characteristic equation are $1.19,1.44,1.44,1.56,1.56$, and 1.05 . The minimum root is 1.05, which is greater than 1.0. In both cases, the minimum roots both are greater than 1.0. In other words, $C_{\varepsilon}$ is greater than 1.0; thus, the crack behavior was stable in 2002.

According to the above analysis method, the minimum roots from 2003 to 2008 are calculated, and results are shown in Figure 10 (the minimum root in 2002 is 1.05). The minimum roots from 2002 to 2008 have some fluctuations, but they are all outside the unit circle, which means they are always greater than 1.0. It indicates that although the development trend of crack behavior fluctuated, the crack behavior was stable. The conclusion is consistent with that in literature [39], which was analyzed according to technical 


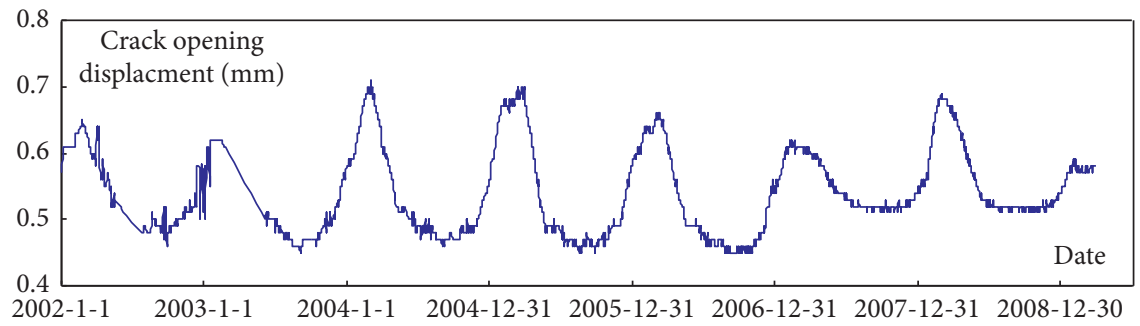

FIGURE 2: Measured data curve of crack opening displacement.

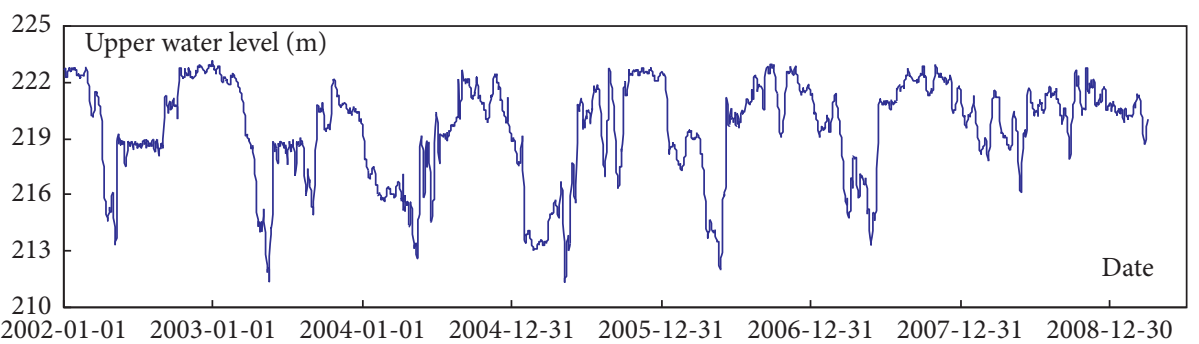

FIgURE 3: Variation curve of the measured upper water level of the dam.

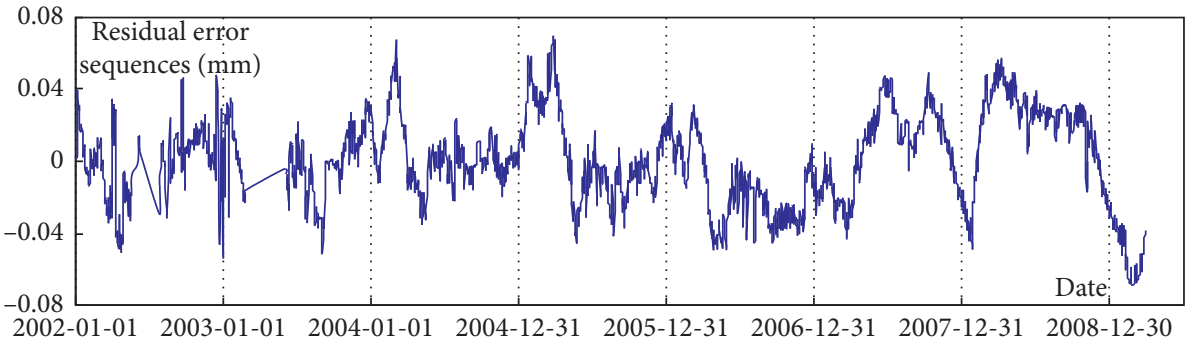

FIgURE 4: The residual sequences of monitoring models.
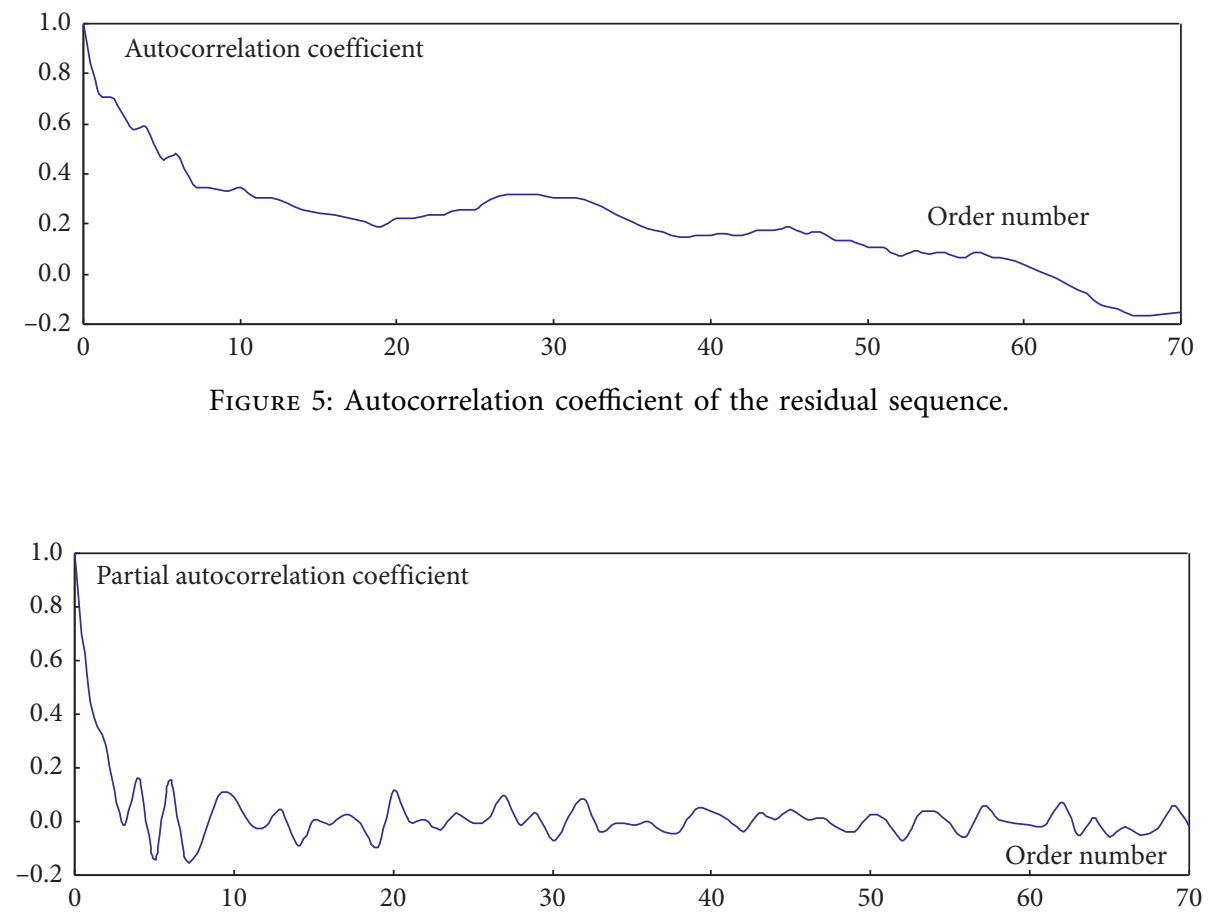

Figure 6: Partial autocorrelation coefficient of the residual sequence. 


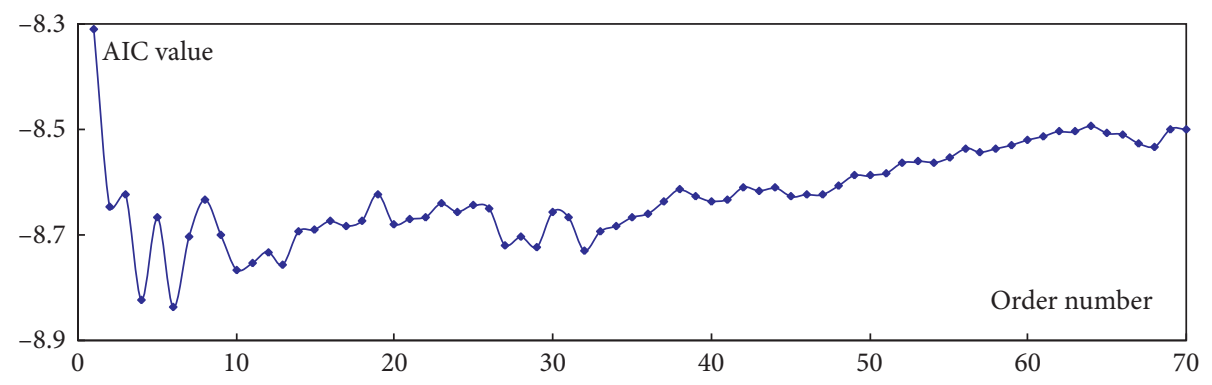

FIgURE 7: Relationship between AIC values and $p$.

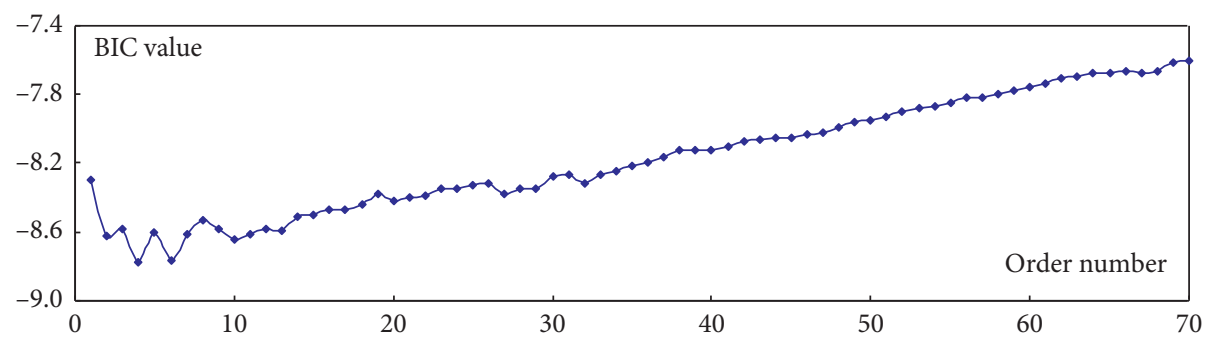

FIGURE 8: Relationship between BIC values and $p$.

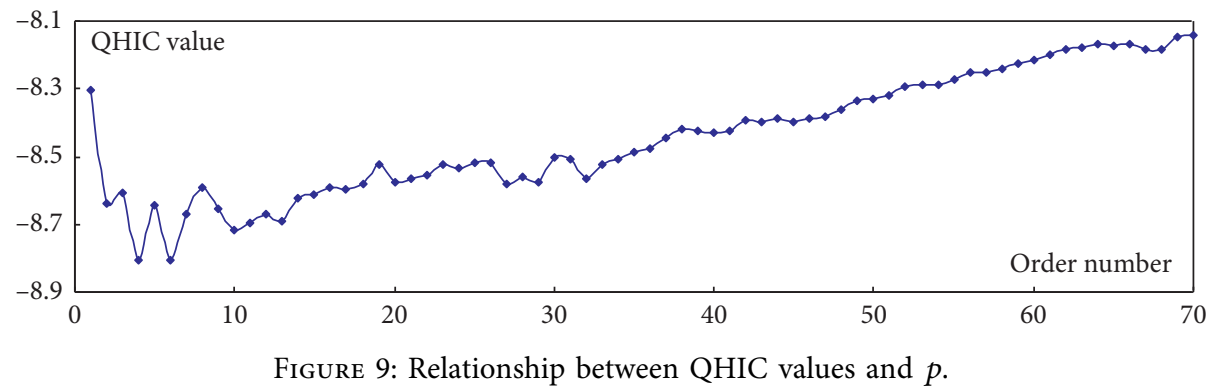

TABLE 1: Calculation results of characteristic roots and the minimum root.

\begin{tabular}{lccccccc}
\hline Order number & 1 & 2 & 3 & 4 & 5 & 6 & $C_{\varepsilon}$ \\
\hline 4 & 1.56 & 1.94 & 1.94 & 1.07 & - & - \\
6 & 1.19 & 1.44 & 1.44 & 1.56 & 1.56 & 1.05 \\
\hline
\end{tabular}

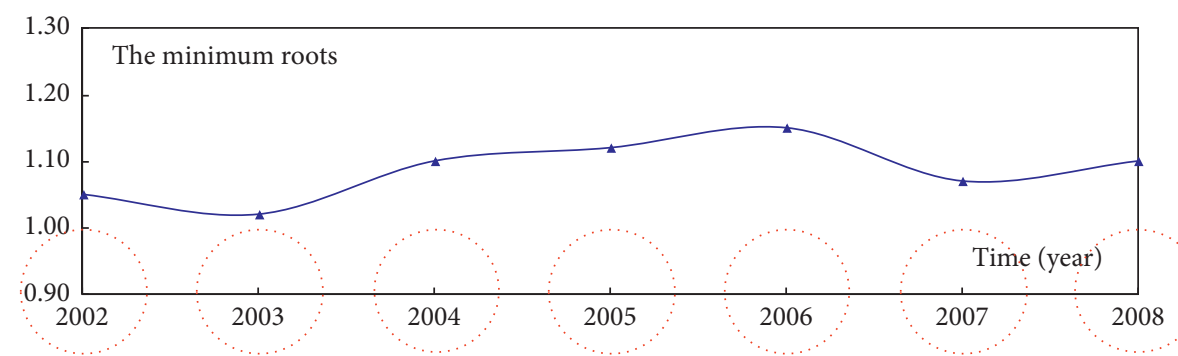

FIGURE 10: Variation curve of the minimum roots with time. 
specification for concrete dam safety monitoring of the People's Republic of China.

In conclusion, the proposed unit root analysis method can be applied to analyze the actual crack behavior of the concrete dam, and it is reasonable and feasible.

\section{Conclusions}

In this paper, a unit root analysis method of actual crack behavior of the concrete dam is proposed based on the monitoring data and the autoregression model. And it is applied to analyze the crack behavior of a concrete gravityarc dam. Some conclusions are drawn as follows:

(1) The residual sequence of the crack monitoring model of the concrete dam can reflect the crack behavior. Therefore, the crack behavior of the concrete dam can be analyzed by the residuals of the crack monitoring model.

(2) Monitoring data of crack opening displacement of the concrete dam can be divided according to the operational control conditions or specified time scale, and they are analyzed by segment modeling method; thus, the residual sequences of monitoring models at different periods can be obtained. If the autocorrelation coefficients of the above residual sequences have the trailing property and the partial autocorrelation coefficients have the truncation property, the autoregression model can be used to analyze the crack behavior.

(3) Parameters of the autoregression model are estimated by the Yule-Walker estimation method, and the order number of autoregression model of the residual sequence of crack monitoring model is determined according to AIC criterion, BIC criterion, and HQIC criterion together. The autoregression model can be established after the order number is determined; thus, the characteristic equation of the autoregression model is obtained. Besides, the characteristic roots of the characteristic equation can be calculated.

(4) The distance between the characteristic roots of the autoregression model and the unit circle can reflect the crack behavior. Short distance represents a bad state, while long distance reflects a good state. Thus, development of crack behavior can be disclosed by variations of characteristic roots of the autoregression models of residual sequences of crack monitoring models at different times.

(5) The case study shows that the unit root analysis method of actual crack behavior is relatively simple and convenient in practical application, which is an available method to evaluate crack behavior based on data in dam health monitoring.

(6) The application of the unit root analysis method of actual crack behavior depends on the crack monitoring models of the concrete dam. Therefore, the influence of different monitoring models on the results of the unit root analysis method of actual crack behavior can be studied in the future.

\section{Data Availability}

The data used to support the findings of this study are included within the article.

\section{Conflicts of Interest}

The authors declare that they have no conflicts of interest regarding the publication of this paper.

\section{Acknowledgments}

This research was supported by the National Natural Science Foundation of China (Grant nos. 51409227 and 51779215), Natural Science Foundation of Jiangsu Province (Grant no. BK20171288), Jiangsu Planned Projects for Postdoctoral Research Funds (Grant no. 1501115B), and CRSRI Open Research Program (Grant no. CKWV2016386/KY).

\section{References}

[1] Z. Wu, H. Rong, J. Zheng, and W. Dong, "Numerical method for mixed-mode I-ii crack propagation in concrete," Journal of Engineering Mechanics, vol. 139, no. 11, pp. 1530-1538, 2013.

[2] N. Sukumar, D. L. Chopp, and B. Moran, "Extended finite element method and fast marching method for three-dimensional fatigue crack propagation," Engineering Fracture Mechanics, vol. 70, no. 1, pp. 29-48, 2003.

[3] G. Zi, H. Chen, J. Xu, and T. Belytschko, "The extended finite element method for dynamic fractures," Shock and Vibration, vol. 12, no. 1, pp. 9-23, 2005.

[4] S. Zhang, G. Wang, and X. Yu, "Seismic cracking analysis of concrete gravity dams with initial cracks using the extended finite element method," Engineering Structures, vol. 56, pp. 528-543, 2013.

[5] I. F. Dias, J. Oliver, J. V. Lemos, and O. Lloberas-Valls, "Modeling tensile crack propagation in concrete gravity dams via crack-path-field and strain injection techniques," Engineering Fracture Mechanics, vol. 154, pp. 288-310, 2016.

[6] Y.-J. Chiou, Y.-M. Lee, and R.-J. Tsay, "Mixed mode fracture propagation by manifold method," International Journal of Fracture, vol. 114, no. 4, pp. 327-347, 2002.

[7] J. Pan, C. Zhang, Y. Xu, and F. Jin, "A comparative study of the different procedures for seismic cracking analysis of concrete dams," Soil Dynamics and Earthquake Engineering, vol. 31, no. 11, pp. 1594-1606, 2011.

[8] M. A. Hariri-Ardebili, "Impact of foundation nonlinearity on the crack propagation of high concrete dams," Soil Mechanics and Foundation Engineering, vol. 51, no. 2, pp. 72-82, 2014.

[9] M. A. Hariri-Ardebili and S. M. Seyed-Kolbadi, "Seismic cracking and instability of concrete dams: smeared crack approach," Engineering Failure Analysis, vol. 52, pp. 45-60, 2015.

[10] M. A. Hariri-Ardebili and V. Saouma, "Quantitative failure metric for gravity dams," Earthquake Engineering \& Structural Dynamics, vol. 44, no. 3, pp. 461-480, 2015.

[11] M. A. Hariri-Ardebili, V. E. Saouma, and K. A. Porter, "Quantification of seismic potential failure modes in concrete dams," Earthquake Engineering \& Structural Dynamics, vol. 45, no. 6, pp. 979-997, 2016. 
[12] M. A. Hariri-Ardebili and M. R. Kianoush, "Seismic analysis of a coupled dam-reservoir-foundation system considering pressure effects at opened joints," Structure and Infrastructure Engineering, vol. 11, no. 7, pp. 833-850, 2015.

[13] M. Shi, H. Zhong, E. T. Ooi, C. Zhang, and C. Song, "Modelling of crack propagation of gravity dams by scaled boundary polygons and cohesive crack model," International Journal of Fracture, vol. 183, no. 1, pp. 29-48, 2013.

[14] K. Wang, Q. Zhang, X. Xia, L. Wang, and X. Liu, "Analysis of hydraulic fracturing in concrete dam considering fluidstructure interaction using XFEM-FVM model," Engineering Failure Analysis, vol. 57, pp. 399-412, 2015.

[15] Z. Shi, M. Suzuki, and M. Nakano, "Numerical analysis of multiple discrete cracks in concrete dams using extended fictitious crack model," Journal of Structural Engineering, vol. 129, no. 3, pp. 324-336, 2003.

[16] P. Cong, C. Gu, and J. Wang, "Application of entropy theory in the analysis of concrete crack propagation process," Journal of Basic Science and Engineering, vol. 16, no. 1, pp. 50-56, 2008.

[17] B. Wu, Z. Wu, B. Chen, H. Su, T. Bao, and S. Wang, "Crack status analysis for concrete dams based on measured entropy," Science China Technological Sciences, vol. 59, no. 5, pp. 777-782, 2016.

[18] X. Li, H. Xu, C. Gu, and Z. Wu, "Law of time-dependent deformation of crack based on wavelet and phase space reconstruction technique," Journal of Hydraulic Engineering, vol. 38, no. 2, pp. 250-254, 2007.

[19] C. Bernstone and A. Heyden, "Image analysis for monitoring of crack growth in hydropower concrete structures," Measurement, vol. 42, no. 6, pp. 878-893, 2009.

[20] Z. Li, C. Gu, Z. Wang, and Z. Wu, "On-line diagnosis method of crack behavior abnormality in concrete dams based on fluctuation of sequential parameter estimates," Science China Technological Sciences, vol. 58, no. 3, pp. 415-424, 2015.

[21] Z. Wu, Safety Monitoring Theory and its Application of Hydraulic Structures, Higher Education Press, Beijing, China, 2003.

[22] Z. Li, H. Li, and Q. Liu, "Complete sequence small probability method for concrete dam crack behavior abnormality diagnosis," Disaster Advances, vol. 6, pp. 257-264, 2013.

[23] C. Gu, Z. Li, and B. Xu, "Abnormality diagnosis of cracks in the concrete dam based on dynamical structure mutation," Science China Technological Sciences, vol. 54, no. 7, pp. 1930-1939, 2011.

[24] B. Chen, Z. Wu, J. Liang, and Y. Dou, "Time-varying identification model for crack monitoring data from concrete dams based on support vector regression and the bayesian framework," Mathematical Problems in Engineering, vol. 2017, Article ID 5450297, 11 pages, 2017.

[25] B. Dai, C. Gu, E. Zhao, K. Zhu, W. Cao, and X. Qin, "Improved online sequential extreme learning machine for identifying crack behavior in concrete dam," Advances in Structural Engineering, vol. 22, no. 2, pp. 402-412, 2019.

[26] C. Gu and Z. Wu, Safety Monitoring of Dams and Dam Foundations: Theories and Methods and Their Application, Hohai University Press, Nanjing, China, 2006.

[27] F. Li, Study on Analysis Methods for Dam Safety Monitoring Data, Zhejiang University, Hangzhou, China, 2012.

[28] P. C. B. Phillips, "Time series regression with a unit root," Econometrica, vol. 55, no. 2, pp. 277-301, 1987.

[29] D. A. Dickey, W. R. Bell, and R. B. Miller, "Unit roots in time series models: tests and implications," The American Statistician, vol. 40, no. 1, pp. 12-26, 1986.
[30] P. J. Brockwell and R. A. Davis, Time Series: Theory and Methods, Springer, New York, NY, USA, 2006.

[31] T. J. Ulrych and T. N. Bishop, "Maximum entropy spectral analysis and autoregressive decomposition," Reviews of Geophysics, vol. 13, no. 1, pp. 183-200, 1975.

[32] G. U. Yule, "On a method of investigating periodicities in disturbed series, with special reference to Wolfer's sunspot numbers," Philosophical Transactions of the Royal Society of London Series A, vol. 226, pp. 267-298, 1927.

[33] G. T. Walker, "On periodicity in series of related terms," Proceedings of the Royal Society A: Mathematical, Physical and Engineering Sciences, vol. 131, no. 818, pp. 518-532, 1931.

[34] N. Levinson, "The Wiener (root mean square) error criterion in filter design and prediction," Journal of Mathematics and Physics, vol. 25, no. 1-4, pp. 261-278, 1946.

[35] J. Durbin, "The fitting of time-series models," Revue de l'Institut International de Statistique/Review of the International Statistical Institute, vol. 28, no. 3, pp. 233-244, 1960.

[36] H. Akaike, "A new look at the statistical model identification," IEEE Transactions on Automatic Control, vol. 19, no. 6, pp. 716-723, 1974.

[37] G. Schwarz, "Estimating the dimension of a model," The Annals of Statistics, vol. 6, no. 2, pp. 461-464, 1978.

[38] E. J. Hannan and B. G. Quinn, "The determination of the order of an autoregression," Journal of the Royal Statistical Society: Series B (Methodological), vol. 41, no. 2, pp. 190-195, 1979.

[39] C. Gu and D. Zheng, Analysis Report of Dam Monitoring Data of Yantan Hydropower Station in Guangxi, Hohai University, Nanjing, China, 2009. 